UCRL-ID - 124171

\title{
A Room Temperature \\ END/B-VI, Mod. 3 \\ Cross Section Library
}

\author{
Dermott E. Cullen \\ Lawrence Livermore National Laboratory \\ P.O. Box 808 \\ Livermore, CA 94550
}

March, 1996

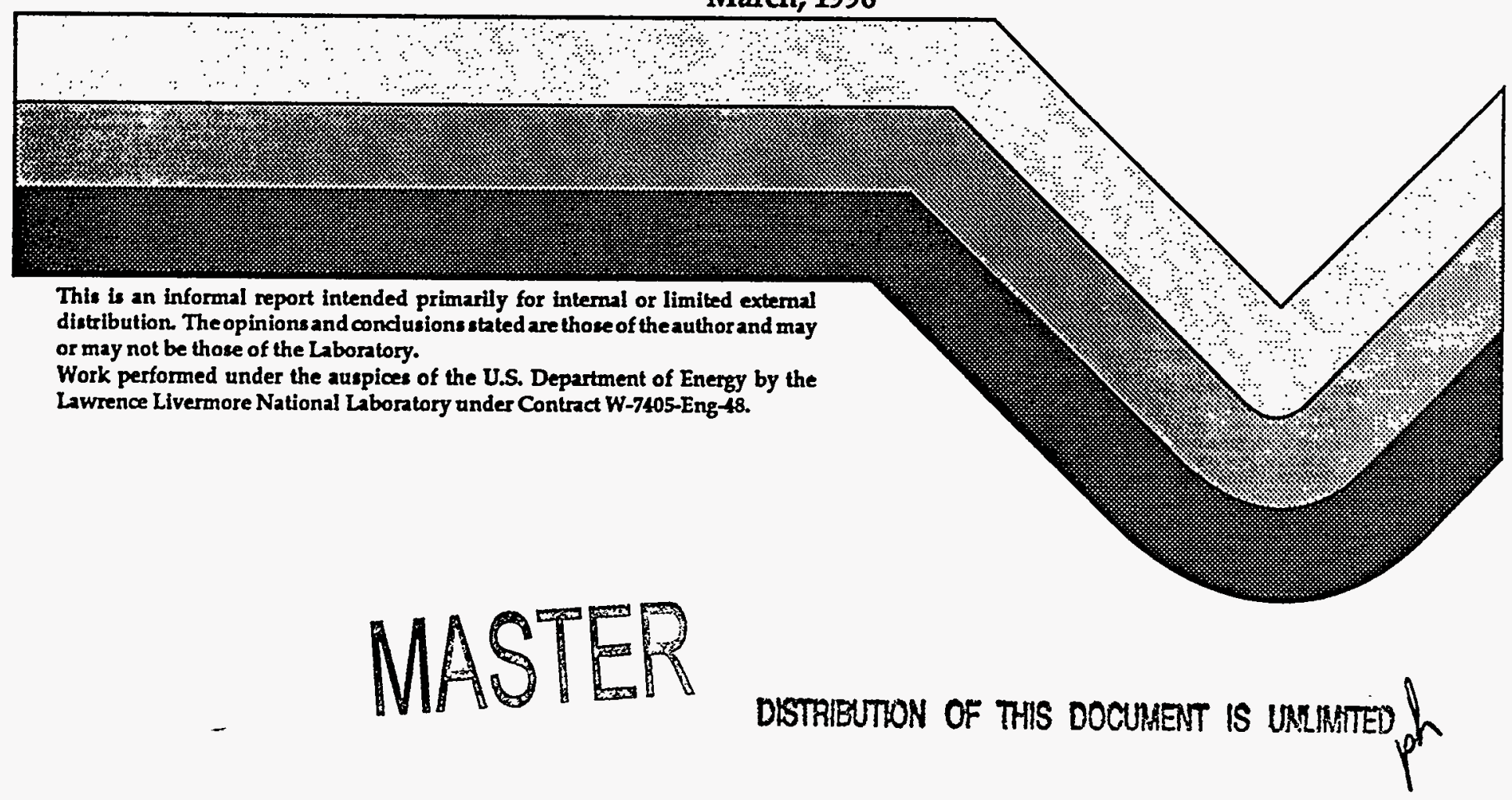




\section{DISCLAIMER}

This document was prepared as an account of work sponsored by an agency of the United States Government. Neither the United States Government nor the University of Califomia nor any of their employees, makes any warranty, express or implied, or assumes any legal liability or responsibility for the accuracy, completeness, or usefulness of any information, apparatus, product, or process disclosed, or represents that its use would not infringe privately owned rights. Reference herein to any specific commercial products, process, or service by trade name, trademark, manufacturer, or otherwise, does not necessarily constitute or imply its endorsement, recommendation, or favoring by the United States Government or the University of California. The views and opinions of authors expressed herein do not necessarily state or reflect those of the United States Government or the University of California, and shall not be used for advertising or product endorsement purposes.

This report has been reproduced directly from the best available copy.

Available to DOE and DOE contractors from the Ollice of Scientific and Technical Information P.O. Box 62, Oak Ridge, TN 37831

Prices available from (615) 576-8401, FTS 626-8401

Available to the public from the National Technical Information Service

U.S. Department of Commerce 5285 Port Royal Rd. Springfield, VA 22161 


\section{DISCLAIMER}

Portions of this document may be illegible in electronic image products. Images are produced from the best available original document. 


\title{
A Room Temperature \\ ENDF/B-VI, Mod. 3 \\ Cross Section Library
}

\author{
b y \\ Dermott E. Cullen \\ University of California \\ Lawrence Livermore National Laboratory \\ P.O. Box 808 \\ L -59 \\ Livermore, CA 94550 \\ tele: $\quad 510-423-7359$ \\ FAX: $\quad 510-422-9560$ \\ E. Mail: cullen1@IInl.gov
}

March 1996

\section{Introduction}

The ENDF/B-VI Mod. 2 library distributed in July 1994 has now been updated to include the most recent modifications. to ENDF/B-VI; namely, Mod. 3. Between the original distribution (Mod. 0) and Mod. 2 libraries, 74 evaluations were updated and distributed in July 1994 (see, UCRL-ID-117797). Between Mod. 2 and 3 libraries, 18 evaluations were updated. No completely new evaluations have been added to the library since the original Mod. 0 release of ENDF/B-VI.

Starting from the original evaluations, including cross sections represented in terms of a combination of resonance parameters and nominally 0 Kelvin cross sections, all of these evaluations have been processed using the ENDF/B Pre-processing codes, to produce room temperature, linearly interpolable tabulated cross sections, in the ENDF/B-VI format, ready for use in applications. 


\section{Data Processing}

The steps required and codes used to produce room temperature, linearly interpolable tabulated cross sections, in the ENDF/B-VI format, are described below (the name of each code in given in parenthesis; for details of éach code, see IAEA-NDS-39, Rev. 8, Nuclear Data Section, International Atomic Energy Agency, Vienna, Austria),

1) Linearly interpolable, tabulated cross sections (LINEAR)

2) Including the resonance contribution (RECENT)

3) Doppler broaden all cross sections to room temperature (SIGMA1)

4) Check data, define redundant cross sections by summation (FIXUP)

The result is linearly interpolable, tabulated, room temperature cross sections, in the ENDF/B-VI format, ready to be used in applications.

\section{Contents of the Library}

This library contains all of the evaluations in the ENDF/B-VI general purpose library. The following page summarizes the contents of the ENDF/B-VI general purpose library. This library contains evaluations for 321 materials (isotopes or naturally occurring elemental mixtures of isotopes).

This library does not contain data from special purpose ENDF/B-VI libraries, such as fission products, thermal scattering, photon interaction data. To obtain any of these special purpose libraries contact the National Nuclear Data Center, Brookhaven National Laboratory.

Each evaluation is stored as a separate file. The following table defines each material and the corresponding filename. The entire library is in the computer independent ENDF/B-VI character format, which allows the data to be easily transported between computers. The entire library requires approximately 132 mega-bytes of storage.

This library contains data for some metastable materials, which are indicated by an " $\mathrm{m}$ " at the end of their descriptions. The library also contains two evaluations for $26-\mathrm{Fe}-56$; a standard evaluation 
extending up to an incident neutron energy of $20 \mathrm{MeV}$, and a second high energy evaluation extending up to $1 \mathrm{GeV}$; the latter is identified in the following table as 26-Fe-56x.

The majority of these evaluations are complete, in the sense that they include all cross sections over the energy range $10^{-5} \mathrm{eV}$ to at least $20 \mathrm{MeV}$. However, the following are only partial evaluations that either only contain single reactions and no total cross section (12-Mg-24, 19-K-41, 22-Ti-46, 22-Ti-47, 22-Ti-48, 22-Ti-50 and 28$\mathrm{Ni}-59$ ), or do not include cross sections above the resonance region (18-Ar-40, 42-Mo-92, 42-Mo-98, 42-Mo-100, 49-In-115, 50-Sn$120,50-\mathrm{Sn}-112$ and $50-\mathrm{Sn}-124)$.

\section{Summary of September 1993 Update}

The library was updated to include the effects of a convention regarding the definition of resolved resonance parameters used by evaluators that was not clearly described in the ENDF/B-VI Formats and Procedures manual. These changes only effect the elastic scattering cross section of the following materials: Fe-54, Fe-56 and $\mathrm{Ni}-58$.

\section{Summary of July 1994 Update}

The 74 evaluations modified between the original (Mod. 0) and Mod. 2 libraries were included in the distribution; Mod. 2 results replaced the older results. For details see the following table of materials modified between the original and Mod. 2 libraries.

\section{Summary of March 1996 Update}

The 18 evaluations modified between Mod. 2 and 3 libraries are included in the current distribution; Mod. 3 results replaced the older results. For details see the following table of materials modified between Mod. 2 and 3.

\section{Acknowledgments}

I thank the Nuclear Data Section of the International Nuclear Energy Agency, Vienna, Austria, for providing the computer time and programmer support to process all of the Mod. 3 data, as they have processed all earlier versions of this library. 
ENDF/B-VI Mod. 3 Library $(\mathrm{Z}=1$ through 51)

\begin{tabular}{|c|c|c|c|c|}
\hline Mater & :ial & ilename & Material & Filename \\
\hline $1-\mathrm{H}$ & -1 & za001001 & $29-\mathrm{Cu}-65$ & za029065 \\
\hline $1-\mathrm{H}$ & -2 & $z a 001002$ & 31-Ga-Nat & $\mathrm{za} 031000$ \\
\hline $1-\mathrm{H}$ & -3 & a001003 & $32-\mathrm{Ge}-72$ & 32072 \\
\hline $2-\mathrm{He}$ & -3 & 02003 & $32-\mathrm{Ge}-73$ & 32073 \\
\hline 2-He & $=-4$ & 02004 & $32-\mathrm{Ge}-74$ & 032074 \\
\hline $3-\mathrm{Li}$ & -6 & 3006 & $32-\mathrm{Ge}-76$ & 32076 \\
\hline $3-\mathrm{Li}$ & -7 & 3007 & $33-A s-75$ & 33075 \\
\hline $4-\mathrm{Be}$ & -9 & 4009 & $34-5 e-74$ & 034074 \\
\hline $5-B$ & -10 & 010 & $34-S e-76$ & 9034076 \\
\hline $5-B$ & -11 & 5011 & $34-5 e-77$ & 34077 \\
\hline $6-c$ & - Nat & 6000 & $34-5 e-78$ & 34078 \\
\hline $7-N$ & -14 & 014 & $34-S e-80$ & 080 \\
\hline $7-N$ & -15 & 7015 & $34-S e-82$ & 34082 \\
\hline $8-0$ & -16 & 016 & $35-B r-79$ & 5079 \\
\hline $8-0$ & -17 & 017 & $35-B r-81$ & 081 \\
\hline $9-E$ & -19 & 9019 & $36-\mathrm{Kr}-78$ & 36078 \\
\hline $11-\mathrm{Na}$ & -23 & 023 & $36-K r-80$ & 36080 \\
\hline $12-\mathrm{Mg}$ & $y-N a t$ & 000 & $36-K r-82$ & 082 \\
\hline $12-\mathrm{Mg}$ & -24 & 2024 & $36-K r-83$ & 36083 \\
\hline $13-\mathrm{A}]$ & -27 & 027 & $36-\mathrm{Kr}-84$ & 084 \\
\hline $14-S i$ & -Nat & 4000 & $36-K r-85$ & 6085 \\
\hline $15-P$ & -31 & 5031 & $36-K x-86$ & 36086 \\
\hline $16-S$ & -Nat & 16000 & $37-R b-85$ & 085 \\
\hline $16-S$ & -32 & 6032 & $37-R b-86$ & 086 \\
\hline $17-\mathrm{CI}$ & -Nat & 000 & $37-\mathrm{Rb}-87$ & 87 \\
\hline $18-\mathrm{Ar}$ & $=-40$ & 18040 & $38-S r-84$ & 38084 \\
\hline $19-K$ & -Nat & 9000 & $38-\operatorname{sr}-86$ & 38086 \\
\hline $19-K$ & -41 & 9041 & $38-\operatorname{sr}-87$ & 087 \\
\hline $20-\mathrm{Ca}$ & -Nat & 20000 & $38-\operatorname{Sr}-88$ & 38088 \\
\hline $21-S c$ & $=-45$ & 1045 & $38-S r-89$ & 8089 \\
\hline 22-Ti & $i-N a t$ & 22000 & $38-S r-90$ & 38090 \\
\hline 22-Ti & $i-46$ & 22046 & $39-Y-89$ & 39089 \\
\hline $22-T i$ & -47 & 2047 & $39-Y-90$ & 090 \\
\hline 22-Ti & $i-48$ & 22048 & $39-Y-91$ & 39091 \\
\hline 22-Ti & $i-50$ & 22050 & $40-\mathrm{Zr}-\mathrm{Nat}$ & 10000 \\
\hline $23-V$ & -Nat & 23000 & $40-2 x-90$ & 40090 \\
\hline $24-\mathrm{Cr}$ & $c-50$ & 24050 & $40-z r-91$ & 40091 \\
\hline $24-\mathrm{Cr}$ & $c-52$ & 4052 & $40-z x-92$ & 0092 \\
\hline $24-\mathrm{Cr}$ & $c-53$ & 24053 & $40-z r-93$ & 10093 \\
\hline $24-\mathrm{Cr}$ & $c-54$ & 4054 & $40-Z r-94$ & 10094 \\
\hline $25-\mathrm{Mn}$ & $1-55$ & 25055 & $40-Z r-95$ & 40095 \\
\hline $26-\mathrm{Fe}$ & -54 & 26054 & $40-z r-96$ & 40096 \\
\hline 26-Fe & -56 & 026056 & $41-N b-93$ & 11093 \\
\hline $26-\mathrm{Fe}$ & $-56 x$ & $026056 x$ & $41-\mathrm{Nb}-94$ & 041094 \\
\hline $26-\mathrm{Fe}$ & -57 & 026057 & $41-N b-95$ & 41095 \\
\hline 26-Ee & -58 & 026058 & 42-Mo-Nat & 42000 \\
\hline 27-Co & -59 & 027059 & $42-\mathrm{MO}-92$ & 042092 \\
\hline $28-\mathrm{Ni}$ & $i-58$ & 028058 & $42-\mathrm{MO}-94$ & 042094 \\
\hline $28-\mathrm{Ni}$ & $i-59$ & 028059 & $42-\mathrm{MO}-95$ & 042095 \\
\hline $28-\mathrm{Ni}$ & $i-60$ & 028060 & $42-M o-96$ & 42096 \\
\hline $28-\mathrm{Ni}$ & $i-61$ & 028061 & $42-\mathrm{MO}-97$ & 042097 \\
\hline $28-\mathrm{Ni}$ & $i-62$ & 028062 & $42-\mathrm{Mo}-98$ & 042098 \\
\hline $28-\mathrm{Ni}$ & $i-6$ & 028064 & $42-\mathrm{MO}-99$ & 042099 \\
\hline $29-c$ & & 029063 & $42-\mathrm{Mo}-100$ & 042100 \\
\hline
\end{tabular}

Material 43-TC-99 44-Ru-96 44-Ru-98 44-Ru-99 44-Ru-100 $44-\mathrm{Ru}-101$ 44-Ru-102 $44-R u-103$ 44-Ru-104 44-Ru-105 44-Ru-106 $45-\mathrm{Rh}-103$ 45-Rh-105 46-Pd-102 46-Pd-104 $46-\mathrm{Pd}-105$ 46-Pd-106 46-Pd-107 46-Pd-108 46-Pd-110 47-Ag- 107 47-Ag-109 $47-\mathrm{Ag}-111$ 48-Cd-Nat 48-Cd-106 $48-\mathrm{Cd}-108$ $48-\mathrm{Cd}-110$ $48-\mathrm{Cd}-111$ $48-\mathrm{Cd}-112$ 48-Cd- 113 48-Cd-114 48-Cd-115m 48-Cd-116 49-In-Nat 49-In-113 49-In-115 50-Sn-112 50-Sn-114 50-Sn-115 50-Sn-116 50-Sn-117 50-Sn-118 50-Sn-119 $50-S n-120$ 50-Sn-122 50-Sn-123 50-Sn-124 $50-S n-125$ 50-Sn-126 51-Sb-121 51-Sb-123 $51-\mathrm{Sb}-124$ 51-Sb-125 51-Sb-126
Filename za043099 za044096 za044098 za044099 za044100 za044101 za044102 za044103 za044104 za044105 za044106 za045103 za045105 za046102 za046104 za046105 za046106 za046107 za046108 za046110 za047107 za047109 za047111 za048000 za048106 za048108 za048110 za 048111 za048112 za048113 za048114 za $048115 \mathrm{~m}$ za0 04116 za049000 za049113 za049115 za050112 za050114 za050115 za050116 za050117 za050118 za050119 za050120 za050122 za050123 za050124 za050125 za050126 za051121 za051123 za051124 za051125 za051126 
ENDF/B-VI Mod. 3 Library ( $Z=52$ through 99)

\begin{tabular}{|c|c|c|c|c|c|}
\hline erial & Filename & rial & me & Material & name \\
\hline $52-\mathrm{Te}-120$ & za052120 & $60-\mathrm{Nd}-147$ & za060147 & $74-W-182$ & za074182 \\
\hline $52-\mathrm{Te}-122$ & za052122 & $60-N d-148$ & za060148 & $74-w-183$ & $2 a 074183$ \\
\hline $52-\mathrm{Te}-123$ & za052123 & $60-N d-150$ & za060150 & $74-W-184$ & $2 a 074184$ \\
\hline $52-\mathrm{Te}-124$ & za052124 & $61-\mathrm{Pm}-147$ & za061147 & $74-W-186$ & $z a 074186$ \\
\hline $52-\mathrm{Te}-125$ & za052125 & $61-\mathrm{Pm}-148$ & za061148 & $75-\mathrm{Re}-185$ & 0075185 \\
\hline $52-\mathrm{Te}-126$ & $z a 052126$ & $61-\mathrm{Pm}-148 \mathrm{~m}$ & za061148m & $75-\operatorname{Re}-187$ & za075187 \\
\hline $52-\mathrm{Te}-127 \mathrm{~m}$ & $\mathrm{za} 052127 \mathrm{~m}$ & $61-P m-149$ & za061149 & $79-\mathrm{Au}-197$ & za079197 \\
\hline $52-\mathrm{Te}-128$ & za052128 & $61-\mathrm{Pm}-151$ & za061151 & $82-P b-206$ & za082206 \\
\hline $52-\mathrm{Te}-129 \mathrm{~m}$ & $\mathrm{za} 052129 \mathrm{~m}$ & $62-\mathrm{Sm}-144$ & za062144 & $82-\mathrm{Pb}-207$ & 2082207 \\
\hline $52-\mathrm{Te}-130$ & $z a 052130$ & $62-\mathrm{Sm}-147$ & $\mathrm{za} 062147$ & $82-\mathrm{Pb}-208$ & $: 0082208$ \\
\hline $52-\mathrm{Te}-132$ & za052132 & $62-\mathrm{sm}-148$ & za062148 & $83-3 i-209$ & za083209 \\
\hline $53-I-127$ & za053127 & $62-\mathrm{Sm}-149$ & za062149 & $90-T h-230$ & : 090230 \\
\hline $53-I-129$ & za053129 & $62-\mathrm{Sm}-150$ & za062150 & $90-\mathrm{Th}-232$ & 090232 \\
\hline $53-I$ & 130 & $62-\mathrm{Sm}-151$ & za062151 & $91-\mathrm{Pa}-231$ & 091231 \\
\hline $53-I-131$ & za053131 & $62-\mathrm{Sm}-152$ & za062152 & $91-P a-233$ & 091233 \\
\hline $53-I-135$ & za053135 & $62-\mathrm{sm}-153$ & za062153 & $92-U-232$ & za092232 \\
\hline $54-X e-124$ & za054124 & $62-\mathrm{Sm}-154$ & za062154 & $92-U-233$ & 233 \\
\hline $54-X e-126$ & za054126 & $63-E u-151$ & za063151 & $92-U-234$ & za092234 \\
\hline $54-X e-128$ & za054128 & $63-E u-152$ & za063152 & $92-\mathrm{U}-235$ & za092235 \\
\hline 129 & za054129 & $63-E u-153$ & za063153 & $92-U-236$ & 20092236 \\
\hline $54-X e-130$ & za054130 & $63-E u-154$ & $2 a 06$ & $92-U-237$ & 237 \\
\hline $54-X e-131$ & za054131 & $63-E u-155$ & zao & $92-U-238$ & 2238 \\
\hline $54-\mathrm{Xe}$ & $2 a 054132$ & $63-E u-156$ & za063156 & $93-N p-237$ & za093237 \\
\hline $54-X e-133$ & za054133 & $63-E u-157$ & za063157 & $93-N p-238$ & za093238 \\
\hline $54-X e-134$ & $z a 054134$ & $64-G d-152$ & za064152 & $93-\mathrm{Np}-239$ & 3239 \\
\hline $54-x$ & za & $64-G d-154$ & $2 a 064154$ & $94-P u-236$ & $2 a 094236$ \\
\hline $54-x$ & za054136 & $64-G d-155$ & za064155 & $94-\mathrm{pu}-237$ & za094237 \\
\hline $55-\mathrm{Cs}-133$ & za05 & $64-G d-156$ & za064156 & $94-\mathrm{Pu}-238$ & za094238 \\
\hline $55-c$ & zao & $64-G d-157$ & $z a 064157$ & $94-P u-239$ & 20094239 \\
\hline $55-c$ & 135 & $64-G$ & za064158 & $94-P u-240$ & $z a 094240$ \\
\hline $55-C s-136$ & za055136 & $64-G d-160$ & za064160 & $94-P u-24 I$ & $z a 094241$ \\
\hline $55-C$ & zao & -159 & zao & $94-P u-242$ & 4242 \\
\hline $56-B$ & zao & $65-\mathrm{Tb}-160$ & 200 & $94-\mathrm{Pu}-243$ & $2 a 094243$ \\
\hline $56-\mathrm{Ba}-135$ & za056135 & $66-D y-160$ & za066160 & $94-\mathrm{Pu}-244$ & za094244 \\
\hline $56-\mathrm{Ba}$ & za056136 & $66-D y-161$ & za066161 & $95-\mathrm{Am}-241$ & za095241 \\
\hline $56-B$ & $\mathrm{za} 0$ & $66-D_{Y}-162$ & 162 & $95-A m-242$ & 242 \\
\hline $56-\mathrm{Ba}$ & 38 & $66-D y-163$ & zao & $95-A m-242 m$ & 242 \\
\hline $56-\mathrm{E}$ & 40 & $66-D y-164$ & zao & $95-A m-243$ & $2 a 095243$ \\
\hline $57-I$ & za057139 & $67-\mathrm{HO}-165$ & $2 a 067165$ & $96-\mathrm{Cm}-241$ & $2 a 096241$ \\
\hline $57-I$ & zao & $68-E x-166$ & za068166 & $96-\mathrm{Cm}-242$ & za096242 \\
\hline $58-$ & 40 & $68-\operatorname{Er}-167$ & zaO & $96-C m-243$ & 5243 \\
\hline $58-c$ & za058141 & $71-L u-175$ & za071175 & $96-\mathrm{Cm}-244$ & za096244 \\
\hline $58-\mathrm{Ce}-142$ & za058142 & $71-$ Lu-176 & za071176 & $96-\mathrm{Cm}-245$ & za096245 \\
\hline $58-c$ & zao & $72-\mathrm{H}$ & $2 a 072000$ & $96-\mathrm{Cm}-246$ & 246 \\
\hline $58-$ & 144 & $72-\mathrm{H}$ & za072174 & $96-\mathrm{Cm}-247$ & za096247 \\
\hline $59-\mathrm{P}$ & za059141 & $72-\mathrm{Hf}-176$ & za072176 & $96-\mathrm{Cm}-248$ & za096248 \\
\hline $59-P r-142$ & za059142 & $72-\mathrm{H} f-177$ & za072177 & $97-\mathrm{Bk}^{-249}$ & za097249 \\
\hline $59-E$ & zaos & $72-\mathrm{Hf}-178$ & za072178 & $98-C f-249$ & za098249 \\
\hline $60-\mathrm{Nd}-142$ & 0142 & -179 & & $98-C f-250$ & za098250 \\
\hline $60-N$ & $2 a 060143$ & $72-\mathrm{Hf}-180$ & $z a 072180$ & $98-C f-251$ & za098251 \\
\hline $60-\mathrm{Nd}-144$ & a060144 & $73-T a-181$ & za073181 & $98-C f-252$ & za098252 \\
\hline $60-\mathrm{Nd}-145$ & $\mathrm{za} 060145$ & 73-Тa-182 & za073182 & $98-C f-253$ & za098253 \\
\hline $60-N d-146$ & za060146 & 74-W -Nat & za074000 & $99-E s-253$ & za099253 \\
\hline
\end{tabular}




\section{Materials Modified for Mod. 2 Library}

\begin{tabular}{|c|c|c|c|c|c|}
\hline Material & Filename & Material & Filename & Material & 150 \\
\hline $1-\mathrm{H}-1$ & 1001001 & $40-2 r-N a t$ & za040000 & $72-\mathrm{H} f-178$ & a072178 \\
\hline $2-\mathrm{He}-3$ & 002003 & $41-\mathrm{Nb}-93$ & a041093 & $72-\mathrm{H} f-179$ & 179 \\
\hline $3-L i-6$ & 03006 & $R u-101$ & 0044101 & $72-\mathrm{Hf}-180$ & 721 \\
\hline $5-B-10$ & 5010 & $44-R u-102$ & a044102 & 74-W -Nat & 074000 \\
\hline $6-\mathrm{C}-\mathrm{Nat}$ & 06000 & $48-C d-106$ & 20048106 & $9-A u-197$ & 9197 \\
\hline $7-N-14$ & & & & -207 & \\
\hline $1-\mathrm{Na}-23$ & & $d-110$ & & $2-U-235$ & 2235 \\
\hline $1-S c-45$ & & $48-c d-112$ & & $2-U-237$ & 237 \\
\hline 50 & & & & $92-U-238$ & \\
\hline & & & & & \\
\hline-53 & & 2 & & & \\
\hline 54 & & 4 & & & 39 \\
\hline 54 & & $53-I-127$ & & 40 & 240 \\
\hline $26-E$ & 6 & $57-\mathrm{La}-139$ & & 41 & 241 \\
\hline $26-\mathrm{Fe}-57$ & & & & & \\
\hline $26-$ & & & & & \\
\hline $27-$ & & $60-1$ & 17 & $95-z$ & 242 \\
\hline $28-1$ & & $61-\mathrm{Pm}-147$ & $z a$ & $96-c$ & 6245 \\
\hline & & & & & \\
\hline $28-N$ & & $62-5$ & & 96 & 47 \\
\hline & & & & & 250 \\
\hline $28-$ & & $\lambda-155$ & $z a$ & 51 & 51 \\
\hline & & $72-\mathrm{Hf}-174$ & & & \\
\hline & & & & $98-C f-253$ & \\
\hline & & & & & \\
\hline
\end{tabular}

\section{Materials Modified for Mod. 3 Library}

$\begin{array}{cc}\text { Material } & \text { Filename } \\ 1-\mathrm{H}-2 & \text { za001002 } \\ 7-\mathrm{N}-14 & \text { za007014 } \\ 13-\mathrm{Al}-27 & \text { za013027 } \\ 48-\mathrm{Cd}-106 & \text { za048106 } \\ 48-\mathrm{Cd}-108 & \text { za048108 } \\ 48-\mathrm{Cd}-110 & \text { za048110 } \\ 48-\mathrm{Cd}-111 & \text { za048111 } \\ 48-\mathrm{Cd}-112 & \text { za048112 } \\ 48-\mathrm{Cd}-113 & \text { za048113 } \\ 48-\mathrm{Cd}-114 & \text { za048114 } \\ 48-\mathrm{Cd}-116 & \text { za048116 } \\ 56-\mathrm{Ba}-138 & \text { za056138 } \\ 56-\mathrm{Ba}-140 & \text { za056140 } \\ 62-\mathrm{Sm}-144 & \text { za062144 } \\ 83-\mathrm{Bi}-209 & \text { za083209 } \\ 92-\mathrm{U}-235 & \text { za092235 } \\ 94-\mathrm{Pu}-241 & \text { za094241 } \\ 95-\mathrm{Am}-241 & \text { za095241 }\end{array}$

